\title{
Two Polymorphisms in the Fractalkine Receptor CX3CR1 Gene Influence the Development of Atherosclerosis: A Meta-Analysis
}

\author{
Jian Wu, ${ }^{1}$ Rui-Xing Yin, ${ }^{1}$ Quan-Zhen Lin, ${ }^{1}$ Tao Guo, ${ }^{1}$ Guang-Yuan Shi, ${ }^{1}$ Jia-Qi Sun, \\ Shao-Wen Shen, ${ }^{1}$ and Qing $\mathrm{Li}^{2}$ \\ ${ }^{1}$ Department of Cardiology, Institute of Cardiovascular Diseases, The First Affiliated Hospital, \\ Guangxi Medical University, 22 Shuangyong Road, Nanning, Guangxi 530021, China \\ ${ }^{2}$ Department of Internal Medicine, Affiliated Shanghai First People’s Hospital, Shanghai Jiao Tong University, Shanghai 200080, China
}

Correspondence should be addressed to Rui-Xing Yin; yinruixing@sohu.com

Received 16 May 2014; Revised 12 July 2014; Accepted 4 August 2014; Published 26 August 2014

Academic Editor: Luisella Bocchio-Chiavetto

Copyright (C) 2014 Jian Wu et al. This is an open access article distributed under the Creative Commons Attribution License, which permits unrestricted use, distribution, and reproduction in any medium, provided the original work is properly cited.

Background. The associations between the Fractalkine receptor (CX3CR1) gene T280M (rs3732378) and V249I (rs3732379) polymorphisms and atherosclerosis (AS) risk are conflicting. The aim of this meta-analysis was undertaken to assess their associations. Methods. PubMed, Embase, Web of Science, Medline, Cochrane database, and CNKI were searched to get the genetic association studies. All statistical analyses were done with Stata 11.0. Results. Twenty-five articles involving 49 studies were included in the final meta-analysis. The analysis showed that the 280M allele carriers of the CX3CR1 T280M polymorphism decreased the risk of AS and coronary artery disease (CAD) in the heterozygous state but increased the risk of ischemic cerebrovascular disease (ICVD) in the homozygote state. The 249I allele carriers of the CX3CR1 V249I polymorphism decreased the risk of AS and CAD in the heterozygous state. The V249I-T280M combined genotype VITM and IITM also decreased the risk of AS. Conclusions. The present meta-analysis suggests that the CX3CR1 T280M and V249I polymorphisms are associated with the susceptibility to AS. However, the results should be interpreted with caution because of the high heterogeneity in the meta-analysis.

\section{Introduction}

Atherosclerosis (AS) is a multifactorial, progressive disease and a major global cause of morbidity and mortality. Atherosclerosis-related cardiovascular diseases, such as coronary artery disease (CAD), acute myocardial infarction (AMI), and ischemic cerebrovascular disease (ICVD), are the causes of death in almost $50 \%$ of cases in developed countries [1]. The exact mechanisms of AS are indistinct. Many studies indicate that the genetic factors play a significant role in the development of AS [2-5]. Until recently, much attention has been focused on this field, and the association of the Fractalkine (FKN) receptor (CX3CR1) gene polymorphisms and AS has been extensively studied [6-31].

It has recently been accepted that AS is predominantly an inflammatory process $[32,33]$ and begins with a fatty streak, which is made up almost entirely of monocytederived macrophages $[33,34]$. During the process of vascular inflammation, chemokines and adhesive molecules exert a vital role via mediating the activation of inflammatory cells and their aggregation or adhesion to vascular walls [35, 36]. FKN is a special chemotactic factor existing in both membrane-bound and soluble forms [37]; the expression of FKN and its receptor CX3CR1 is upregulated in AS lesions [38-40], and the severity of AS is greatly improved by inhibiting their expression [40-42], suggesting that the FKN/CX3CR1 is closely correlated to AS. Given the crucial role of $C X 3 C R 1$ in the inflammatory process, the mutations in the CX3CR1 may also play a significant role in the development of atherosclerotic diseases. Recently, a number of molecular epidemiological studies have been done to evaluate the associations between the CX3CR1 gene polymorphisms (T280M and V249I) and the risk of atherosclerotic diseases [6-31]. However, the results of different studies are inconsistent, possibly due to small sample sizes in the individual studies. In 2009, Apostolakis et al. [43] performed a meta-analysis 
to evaluate the association between the CX3CR1 T280M and V249I polymorphisms and CAD and demonstrated that the CX3CR1 280M allele was associated with a reduced risk of CAD in the heterozygous state and 249I-280M haplotype had a atheroprotective effect on CAD. However, they just studied the 280M allele and 249I-280M haplotype of CX3CR1 in Caucasians. Considering the meta-analysis only focused on the association of the CX3CR1 polymorphism with the single atherosclerotic disease, we, therefore, performed this meta-analysis of all the studies available now to get a more precise estimation of the associations between the CX3CR1 T280 M (rs3732378) and V249I (rs3732379) polymorphisms and overall AS risk.

\section{Materials and Methods}

2.1. Literature Search and Selection. This meta-analysis followed the Preferred Reporting Items for Systematic Reviews and Meta-analyses (PRISMA) criteria [45]. Eligible literatures published before the end of March 1, 2014, were identified by the search of PubMed, Embase, Web of Science, Medline, Cochrane database, and CNKI. Library uses combinations of the following keywords: "chemokine receptor 1" or "FKN" or "CX3CR1" or "fractalkine" and "polymorphism" or "mutation" or "variant" or "variation" or "genotype" and "coronary artery disease" or "CAD" or "coronary heart disease" or "CHD" or "myocardial infarction" or "MI" or "ischemic cardiovascular disease" or "ischemic cardiovascular events" or "ischemic stroke" or "IS" or "cerebrovascular disease" or "ischemic cerebrovascular events" or "cerebral infarction" or "cerebral ischemia" or "brain infarction" or "carotid artery stenosis" or "CAAD" or "transient ischemic attack" or "TIA" or "peripheral arterial disease" or "PAD" or "peripheral artery occlusive disease" or "PAOD" or "renal artery stenosis" or "RAS" or "retinal artery occlusion" or "RAO" or "aortic aneurysm" or "atherosclerosis". In addition, all references cited were reviewed to identify additional studies. If two or more studies sharing the same studied populations, the one with the small size was abandoned. Two reviewers (JW and QZL) searched the above databases independently. Decisions were compared and disagreements about study selection were resolved by involving a third reviewer (RXY). The search was limited to English and Chinese language papers. There was no restriction on time period, sample size, or population.

2.2. Inclusion and Exclusion Criteria. To be included in the present meta-analysis, the studies had to comply with the following major criteria: (1) case-control or cohort studies evaluating the associations between the CX3CR1 T280M and V249I polymorphisms and AS risk; (2) published studies with full text articles; (3) sufficient published data for calculating odds ratios (ORs) with their 95\% confidence intervals (CIs); (4) study population being adults; (5) the diagnosis of ischemic heart disease (CAD and AMI) being accorded with the result of coronary angiography, criteria of World Health Organization (WHO), criteria of European Society of Cardiology (ESC), or criteria of American College of Cardiology/American Heart Association (ACC/AHA); the diagnosis of ICVD is accorded with result of computed tomography (CT) or magnetic resonance imaging (MRI); the diagnosis of carotid atherosclerosis (CAA) was assessed by ultrasound color Doppler (USCD), and peripheral arterial disease (PAD) was diagnosed by the following criteria: clinical symptoms of PAD (intermittent claudication, rest pain, or gangrene) accompanied by an ankle-brachial-index and significant stenosis of leg arteries confirmed by FCDS and/or angiography.

Studies were excluded if they were (1) review or metaanalysis; (2) not conducted in humans; (3) duplicate studies.

2.3. Data Extraction. Data, including name of the first author, year of publication, study population (country, ethnicity), study type (case-control and cohort study), type of atherosclerotic disease, source of controls (populationbased studies and hospital-based studies), sample size (total numbers of cases and controls), and number of genotypes in cases and controls, were extracted from each study by two reviewers independently (JW and QZL) according to the prespecified inclusion criteria. Studies that reported a comparison of two case samples, such as different age groups, were included in the meta-analysis as independent studies. Decisions were compared and disagreements about study selection were resolved by consensus or by involving a third reviewer (RXY).

2.4. Quality Assessment for Individual Studies. The quality of the individual studies was evaluated and scored by two reviewers independently based on the Newcastle-Ottawa Scale (NOS) [46]. Each study was assessed based on three broad perspectives: selection, comparability, and exposure, and each satisfactory answer received one point. The NOS ranges between zero (none of the quality criterion was met) up to nine stars (all the quality criteria were met), and the high-quality study was considered as the one with a score higher than six. The reviewer (RXY) examined the results, and a consensus was reached.

2.5. GRADE Quality Assessment. GRADE (grades of recommendation, assessment, development and evaluation) approach was adopted to grade quality of evidence for each association [47]. The GRADE system included level of evidence: (1) high quality, we are very confident that the true effect lies close to that of the estimate of the effect; (2) moderate quality, we are moderately confident in the effect estimate: the true effect is likely to be close to the estimate of the effect, but there is a possibility that it is substantially different; (3) low quality, our confidence in the effect estimate is limited: the true effect may be substantially different from the estimate of the effect; and (4) very low quality, we have very little confidence in the effect estimate: the true effect is likely to be substantially different from the estimate of effect. Two reviewers (JW and QZL) assessed quality independently and solved disagreement by discussion.

2.6. Statistical Analysis. For the controls of each study, Hardy-Weinberg equilibrium (HWE) was assessed using the chi-square test $(P<0.05$ was considered significant deviation 


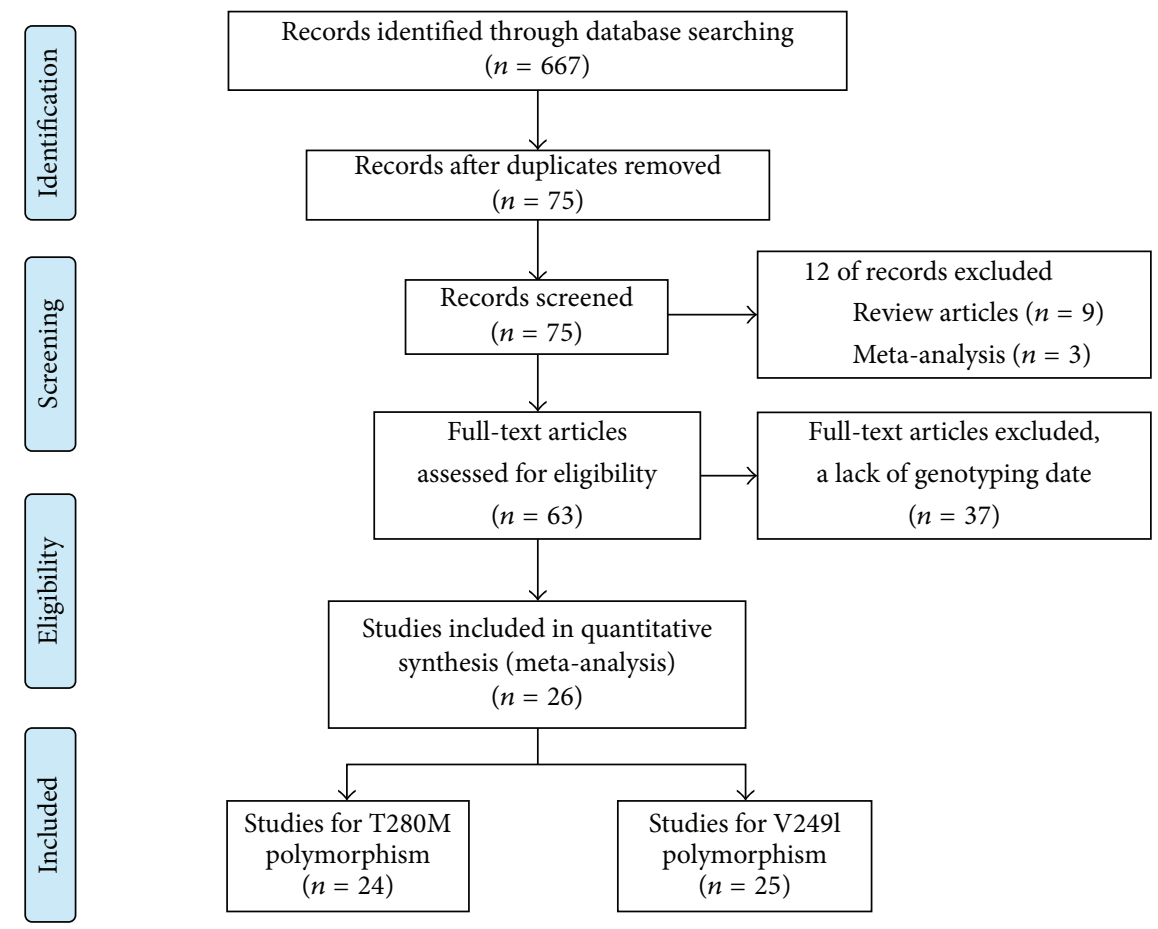

FIGURE 1: Flow chart showing study selection process.

from HWE). We performed a haplotype analysis based on the genotype data, the haplotype frequencies were calculated by CubeX analysis software for each study separately and for the whole sample [48]. The strength of associations between the CX3CR1 T280M and V249I polymorphisms and AS risk was assessed by ORs with $95 \%$ CIs. The pooled ORs were performed for dominant model ( $\mathrm{T} / \mathrm{M}+\mathrm{M} / \mathrm{M}$ versus $\mathrm{T} / \mathrm{T}$ for T280M; V/I + I/I versus V/V for V249I), recessive model $(\mathrm{M} / \mathrm{M}$ versus $\mathrm{T} / \mathrm{T}+\mathrm{T} / \mathrm{M}$ for $\mathrm{T} 280 \mathrm{M} ; \mathrm{I} / \mathrm{I}$ versus $\mathrm{V} / \mathrm{V}+\mathrm{V} / \mathrm{I}$ for V249I), codominant model ( $\mathrm{T} / \mathrm{M}$ versus $\mathrm{T} / \mathrm{T}+\mathrm{M} / \mathrm{M}$ for T280M; V/I versus V/V + I/I for V249I), additive model (M/M versus $\mathrm{T} / \mathrm{T}$ for T280M; I/I versus $\mathrm{V} / \mathrm{V}$ for V249I), and allelic model ( $\mathrm{M}$ allele versus $\mathrm{T}$ allele for T280M; I allele versus $\mathrm{V}$ allele for V249I). Bonferroni correction was used to control for the multiple testing in view of five genetic models under investigation (significance was set at $0.05 / 5=0.01$ ), then another statistical significance was set as $P<0.05$.

Heterogeneity across individual studies was calculated using Cochran's $Q$ statistic $\left(P_{\mathrm{Q}}\right)$ and the $I^{2}$ statistic. Values for $P_{Q}<0.10$ and $I^{2}>50 \%$ indicate a presence of heterogeneity among studies $[49,50]$, and the randomeffects model was used for the meta-analysis. Otherwise, the fixed-effects model was used. Subgroup analysis was used to alleviate the heterogeneity based on ethnicity (Asian and Caucasians), the type of disease (CAD, ICVD, CAA, and PAD), and source of controls (population-based and hospitalbased).

Sensitivity analyses were performed based on HWE (studies without HWE were excluded) and NOS score (studies with score $\leq 6$ were excluded). Begg's funnel plot and Egger's regression test were conducted to identify possible publication bias in the current meta-analysis $(P<0.05$ was considered representative of statistically significant publication bias). The analyses were performed using Stata software11.0 (StataCorp LP, College Station, USA).

\section{Results}

3.1. Study Characteristics. The present study met the PRISMA statements and PRISMA flow chart (Checklist S1 available online at http://dx.doi.org/10.1155/2014/913678 and Figure 1). A total of 667 articles were identified after searching. After careful review, 26 articles involving 49 studies (24 studies for T280M and 25 studies for V249I polymorphisms) met the inclusion criteria and were selected in this meta-analysis [6-31]; one duplicate study was cut [18]. For the CX3CR1 T280M polymorphism, 7732 AS cases and 5905 controls were included to assess the association between the variant and AS risk [6-12, 15-19, 21-28, 30, 31]. For the CX3CR1 V249I polymorphism, 7952 AS cases and 6035 controls were included to assess the association between the variant and AS risk [6-12, 15-24, 26-31]. Main characteristics of the included studies were listed in Tables 1 and 2. The most commonly atherosclerotic disease included in the present meta-analysis was CAD in 21 studies. In addition, there were 8 studies involving carotid atherosclerosis, 8 studies involving cerebral infarction, 6 studies involving AMI, 2 studies involving ICVD, 2 studies involving ischemic stroke (IS), and 2 studies involving PAD. There were 24 studies of Asians and 25 studies of Caucasians. Three studies did not follow the HWE $[8,23,26]$. The results of GRADE were shown in Table S1. 


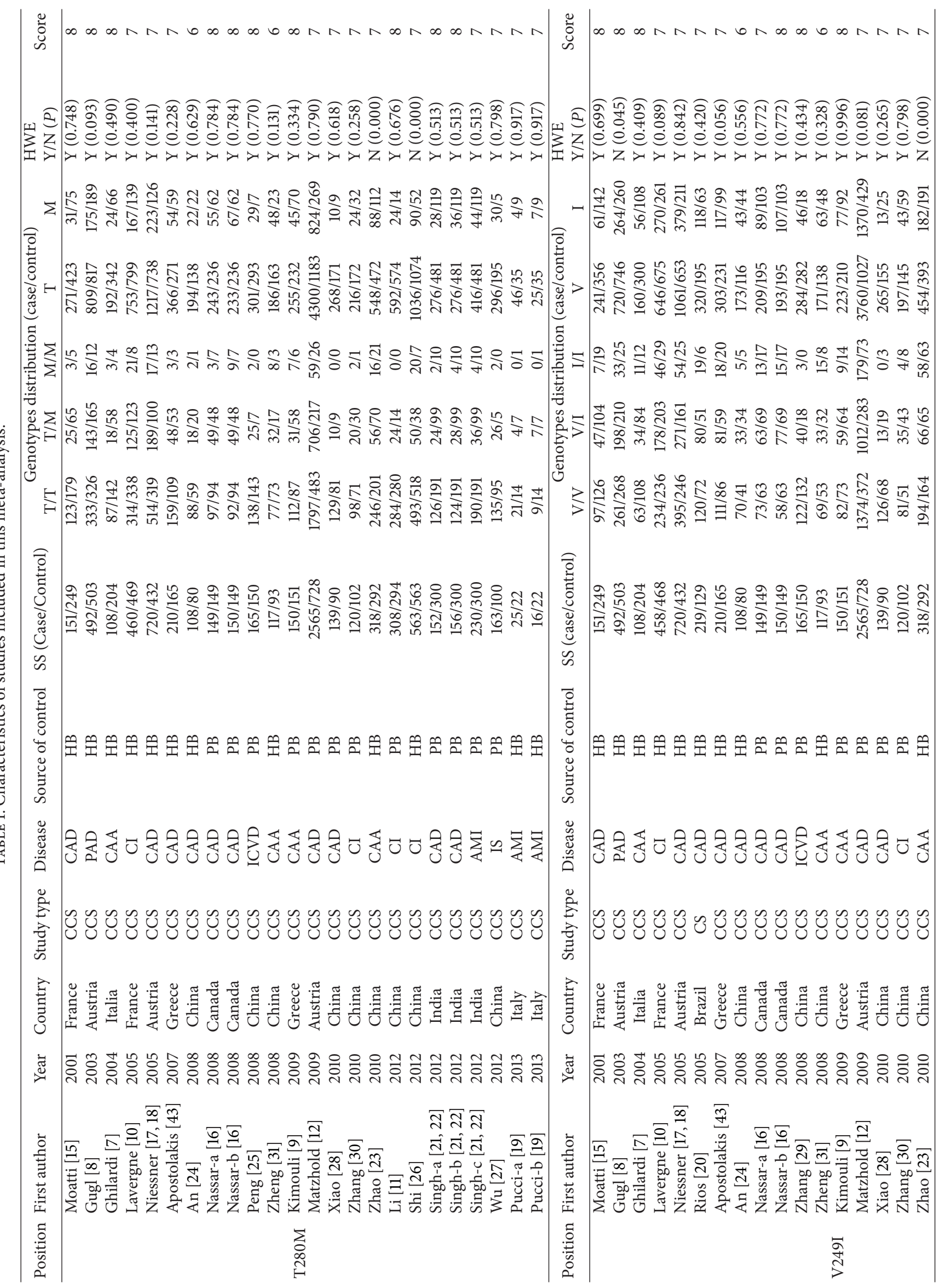




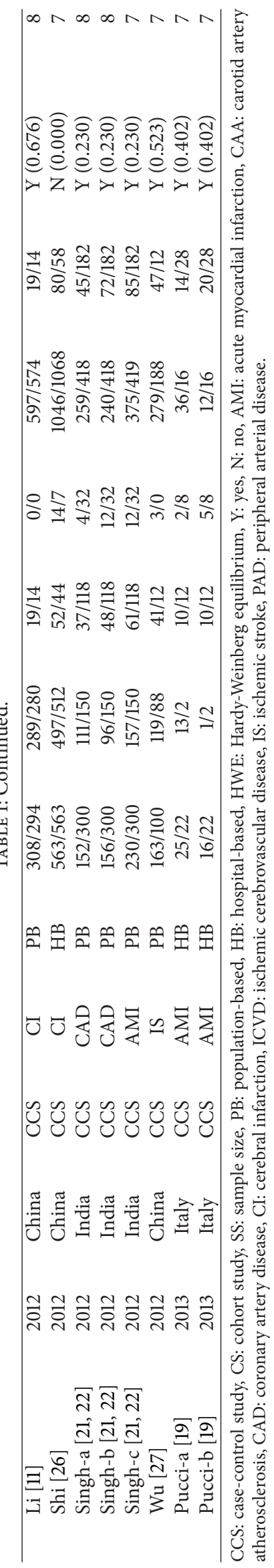




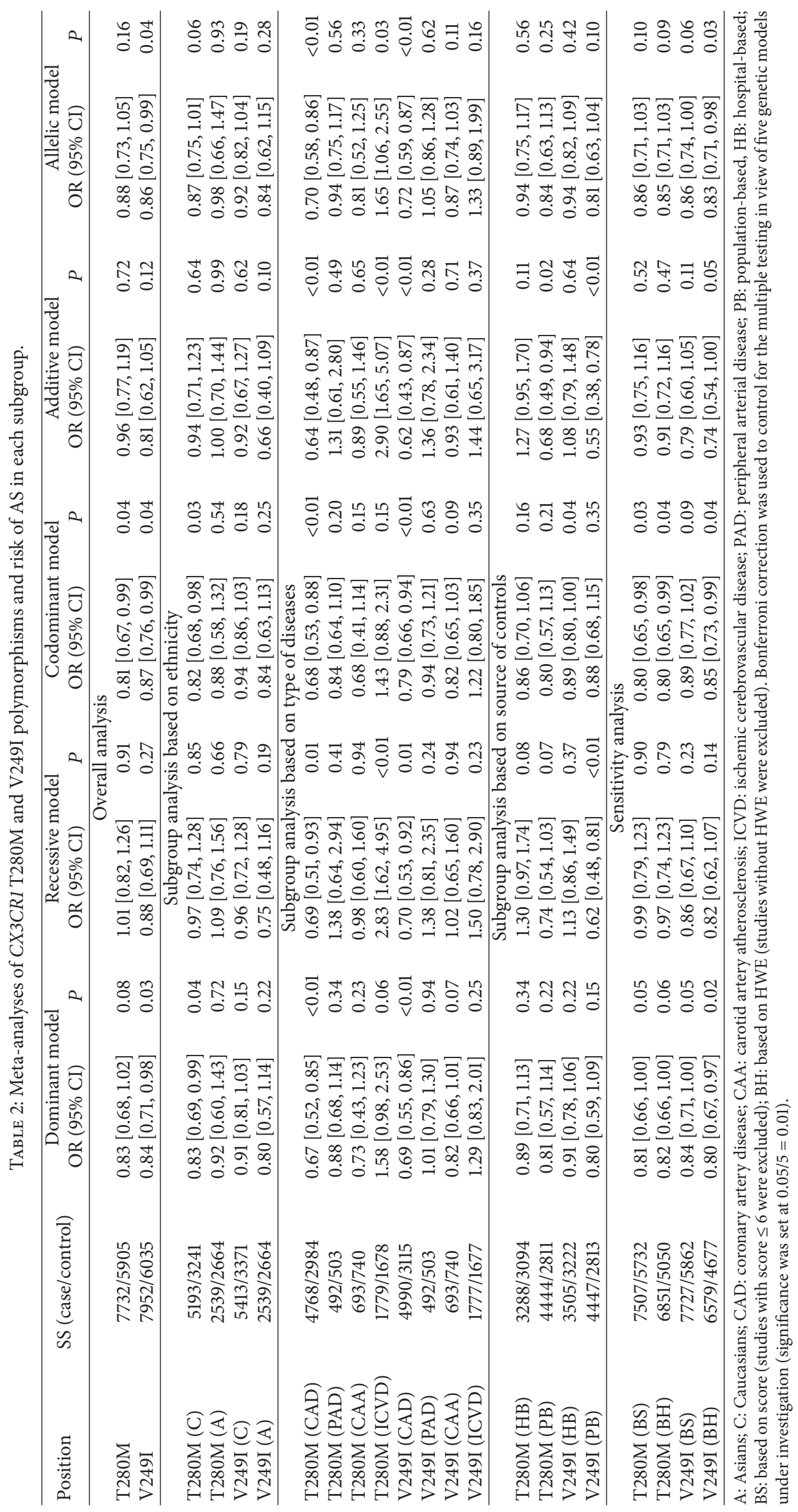




\begin{tabular}{|c|c|c|c|}
\hline Study ID & & OR $(95 \% \mathrm{CI})$ & Weight (\%) \\
\hline Moatti et al. (2001) & & $0.56(0.33,0.94)$ & 4.47 \\
\hline Gugl et al. (2003) & & $0.85(0.65,1.11)$ & 5.70 \\
\hline Ghilardi et al. (2004) & & $0.51(0.28,0.92)$ & 4.08 \\
\hline Lavergne et al. (2005) & & $1.09(0.82,1.47)$ & 5.60 \\
\hline Niessner et al. (2005) & & $1.17(0.89,1.55)$ & 5.66 \\
\hline Apostolakis et al. (2007) & & $0.62(0.39,0.98)$ & 4.76 \\
\hline An et al. (2008) & & $0.90(0.29,1.24)$ & 3.50 \\
\hline Nassar et al. a (2008) & & $0.99(0.61,1.61)$ & 4.61 \\
\hline Nassar et al. b (2008) & & $1.04(0.64,1.70)$ & 4.60 \\
\hline Peng et al. (2008) & $\rightarrow$ & $3.70(1.55,8.83)$ & 2.89 \\
\hline Zheng et al. (2008) & & $1.78(0.91,3.49)$ & 3.71 \\
\hline Kimouli et al. (2009) & & $0.42(0.25,0.70)$ & 4.46 \\
\hline Matzhold et al. (2009) & & $0.87(0.73,1.05)$ & 6.05 \\
\hline Xiao et al. (2010) & & $0.70(0.27,1.79)$ & 2.64 \\
\hline Zhang et al. (2010) & & $0.48(0.25,0.92)$ & 3.84 \\
\hline Zhao et al. (2010) & & $0.65(0.44,0.97)$ & 5.08 \\
\hline Li et al. (2012) & & $1.69(0.86,3.33)$ & 3.67 \\
\hline Shi et al. (2012) & & $1.38(0.89,2.15)$ & 4.87 \\
\hline Singh et al. a (2012) & & $0.37(0.22,0.61)$ & 4.56 \\
\hline Singh et al. b (2012) & & $0.44(0.27,0.70)$ & 4.68 \\
\hline Singh et al. c (2012) & & $0.37(0.24,0.56)$ & 4.91 \\
\hline Wu et al. (2012) & $\rightarrow$ & $3.66(1.36,9.87)$ & 2.48 \\
\hline Pucci et al. a (2013) & & $0.38(0.09,1.55)$ & 1.54 \\
\hline Pucci et al. b (2013) & $\rightarrow$ & $1.56(0.41,5.95)$ & 1.65 \\
\hline Overall $\left(I^{2}=76.2 \%, P=0.000\right)$ & & $0.81(0.66,0.99)$ & 100.00 \\
\hline \multicolumn{4}{|l|}{ Note: weights are from random effects analysis } \\
\hline 1 & 1 & & \\
\hline 0.22 & 2.5 & & \\
\hline
\end{tabular}

FIGURE 2: Forest plot for CX3CR1 T280M polymorphism and AS risk (TM versus TT).

3.2. Genotype-Phenotype Association. As shown in Table 2, the overall results showed no significant association between the CX3CR1 T280M polymorphism and the susceptibility to AS in five genetic models $(P>0.01$ for all). When we performed a subgroup analysis, there was significant association between T280M polymorphism and the susceptibility to AS in the CAD group in dominant model $(P<$ $0.01)$, codominant model $(P<0.01)$, additive model $(P<$ $0.01)$, and allelic model $(P<0.01)$, but not in recessive model $(P>0.01)$, suggesting that the $280 \mathrm{M}$ allele carriers decreased the risk of CAD in the heterozygous state. The significant association between T280M polymorphism and the susceptibility to AS was also found in the ICVD group in recessive model $(P<0.01)$ and additive model $(P<$ $0.01)$, but not in dominant model $(P>0.01)$, codominant model $(P>0.01)$, or allelic model $(P>0.01)$, suggesting that the $280 \mathrm{M}$ allele carriers increased the risk of ICVD in homozygote state. Then, we considered the TT genotype as the baseline risk, we found TM genotype was a protective role for AS $(\mathrm{OR}=0.81,95 \% \mathrm{CI}=0.66-0.99, P=0.04$; Table 3 and Figure 2), subgroup analysis showed that the TM genotype was a protective role for $\mathrm{CAD}(\mathrm{OR}=0.67,95 \% \mathrm{CI}=0.52-$ $0.87, P<0.01$; Table 3 and Figure $\mathrm{S} 1$ ), and MM genotype was a risk factor for ICVD $(\mathrm{OR}=2.88,95 \% \mathrm{CI}=1.64-5.04$, $P<0.001$; Table 3 and Figure S2). There was no association between the T280M polymorphism and the susceptibility to AS in the other groups (Tables 2 and 3).

The overall results showed no significant association between the CX3CR1 V249I polymorphism and the susceptibility to AS in five genetic models ( $P>0.01$ for all, Table 2$)$. Subgroup analysis showed significant association between V249I polymorphism and the susceptibility to AS in CAD group in dominant model $(P<0.01)$, codominant model $(P<0.01)$, additive model $(P<0.01)$, and allelic model $(P<0.01)$, but not in recessive model $(P>0.01)$, suggesting that the 249I alleles decreased the risk of CAD in heterozygote state. Significant associations were also found between this variant and the susceptibility to AS in the population-based 
TABLE 3: The independent effect of each genotype/haplotype on susceptibility to atherosclerosis.

\begin{tabular}{|c|c|c|c|c|c|c|c|c|}
\hline Genotype & Cases $(n)$ & Controls $(n)$ & Baseline risk & OR & $95 \%$ CI & $P$ & $I^{2}(\%)$ & $P_{\mathrm{Q}}$ \\
\hline VVTT & 1923 & 2601 & & 1 & & & & \\
\hline VVTM & 49 & 3 & VVTT & 11.95 & $5.00-28.58$ & $<0.001$ & 0 & 0.44 \\
\hline VITT & 516 & 700 & VVTT & 1.11 & $0.96-1.27$ & 0.16 & 31.4 & 0.13 \\
\hline VITM & 605 & 1211 & VVTT & 0.64 & $0.50-0.82$ & $<0.001$ & 69.2 & $<0.1$ \\
\hline IITT & 64 & 76 & VVTT & 1.20 & $0.83-1.74$ & 0.33 & 0 & 0.46 \\
\hline IITM & 100 & 182 & VVTT & 0.69 & $0.52-0.90$ & $<0.01$ & 41.7 & $<0.1$ \\
\hline IIMM & 87 & 151 & VVTT & 0.76 & $0.57-1.02$ & 0.06 & 0 & 0.79 \\
\hline VV & 4813 & 3556 & & 1 & & & & \\
\hline VI & 2598 & 2016 & VV & 0.84 & $0.72-0.98$ & 0.02 & 65.9 & $<0.1$ \\
\hline VI (CAD) & 1843 & 1227 & VV (CAD) & 0.72 & $0.59-0.90$ & $<0.01$ & 68.1 & $<0.1$ \\
\hline II & 541 & 463 & VV & 0.81 & $0.62-1.05$ & 0.12 & 61.6 & $<0.1$ \\
\hline $\mathrm{TT}$ & 5786 & 4293 & & 1 & & & & \\
\hline $\mathrm{TM}$ & 1743 & 1456 & TT & 0.81 & $0.66-0.99$ & 0.04 & 76.2 & $<0.1$ \\
\hline TM (CAD) & 1193 & 871 & TT (CAD) & 0.67 & $0.52-0.87$ & $<0.01$ & 72.2 & $<0.1$ \\
\hline MM & 203 & 156 & TT & 0.96 & $0.77-1.19$ & 0.72 & 31.9 & 0.08 \\
\hline MM (ICVD) & 47 & 16 & TT (ICVD) & 2.88 & $1.64-5.04$ & $<0.001$ & 0 & 0.92 \\
\hline VT & 5377 & 6975 & & 1 & & & & \\
\hline VM & 77 & 5 & VT & 11.47 & $5.34-24.64$ & $<0.001$ & 43.0 & 0.12 \\
\hline IT & 814 & 1007 & VT & 1.09 & $0.97-1.21$ & 0.14 & 0 & 0.55 \\
\hline $\mathrm{IM}$ & 892 & 1665 & VT & 0.63 & $0.50-0.81$ & $<0.001$ & 82.2 & $<0.1$ \\
\hline
\end{tabular}

$P_{\mathrm{Q}}, P$ value for Cochran's $Q$ statistic.

(PB) group in recessive and additive models $(P<0.01$ for each). Then, we considered the VV genotype as the baseline risk; we found VI genotype was a protective role for AS $(\mathrm{OR}=0.84,95 \% \mathrm{CI}=0.72-0.98, P=0.02$; Table 3 and Figure 3); subgroup analysis showed that the VI genotype was a protective role for $\mathrm{CAD}(\mathrm{OR}=0.72,95 \% \mathrm{CI}=0.59-0.90$, $P<0.01$; Table 3 and Figure S3). There was no association between V249I polymorphism and the susceptibility to AS in the other groups (Tables 2 and 3).

For V249I-T280M combined genotype (Table S2), when we took VVTT genotype as a baseline risk, significant discrepancies were found in VVTM genotype (OR $=11.95$, 95\% CI $=5.00-28.58, P<0.001$; Table 3 ), suggesting that the VVTM genotype was a risk factor for AS, but VVTM was a very rare genotype and may not have potential clinical significance. Significant association was also found in the $\operatorname{VITM}(\mathrm{OR}=0.64,95 \% \mathrm{CI}=0.50-0.82, P<0.001)$ and IITM genotypes $(\mathrm{OR}=0.69,95 \% \mathrm{CI}=0.52-0.90, P<$ 0.01 ), suggesting that the VITM and IITM genotypes were protective roles for AS (Table 3 ).

3.3. Linkage Disequilibrium Analysis. There were nine combined genotypes in Table S2; six studies identified the extremely rare VVTM genotype. Linkage disequilibrium (LD) analysis showed a strong association between the T280M and V249I polymorphisms (Table 4). Thirteen of the fourteen studies conclusively found complete LD; an association analysis of the whole sample (cases and controls) indicated strong (but not complete) LD between the two loci $\left(D^{\prime}=0.9950, r^{2}=0.5394\right)$.

3.4. Haplotype-Phenotype Association. Haplotype frequencies of each study and the total sample are reported in Table 4, the strong LD between the V249I and T280M polymorphisms resulted in three predominant haplotypes and the extremely rare $249 \mathrm{~V}-280 \mathrm{M}$; the latter haplotype accounts for less than $0.5 \%$ of the entire population's gene pool. We assessed the effect of the $249 \mathrm{~V}-280 \mathrm{M}, 249 \mathrm{I}-280 \mathrm{~T}$, and 249I$280 \mathrm{M}$ haplotypes on the risk for AS independently taking the 249V-280T haplotype as a baseline risk. A significant predominance of the 249I-280M haplotype was observed in the control population compared to case subjects $(\mathrm{OR}=$ 0.63, 95\% CI: $0.50-0.81, P<0.001$; Table 3$)$. No association was observed between the 249I-280T haplotype and susceptibility to AS (OR = 1.09, 95\% CI: 0.97-1.21, $P=0.14$; Table 3).

3.5. Sensitivity Analysis. Sensitivity analyses were conducted to determine whether modification of the inclusion criteria of the meta-analysis affected the final results. The included studies were limited to those conforming to HWE or the high quality studies (NOS score $\geq 7$ ); the corresponding pooled ORs were not materially altered, either for the CX3CR1 T280M polymorphism or for the V249I polymorphism. This suggested that the overall results of this meta-analysis were statistically robust. The main results of sensitivity analyses are shown in Table 2.

3.6. Heterogeneity Analysis. For both CX3CR1 T280M and V249I polymorphisms, significant heterogeneity existed in the overall comparisons in five genetic models. We performed subgroup analyses based on the ethnic (Caucasians and Asian), atherosclerotic diseases (CAD, PAD, CAA, and ICVD), and source of control (hospital-based or populationbased); heterogeneity was distinctly reduced; although it was 


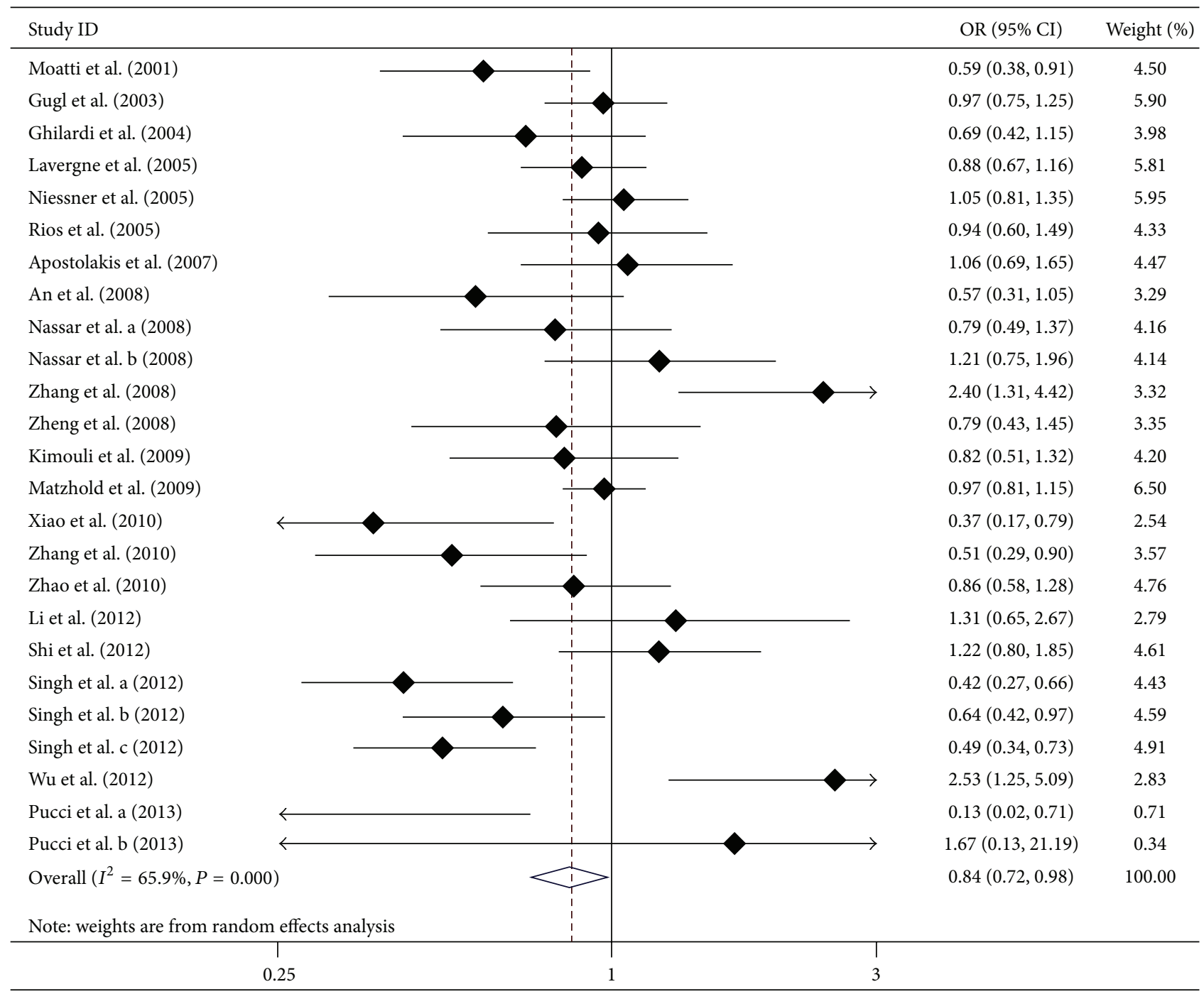

FIGURE 3: Forest plot for CX3CR1 V249I polymorphism and AS risk (VI versus VV).

still significant, we could not point out other possible sources of heterogeneity. The data were shown in Table S3.

For the V249I-T280M combined polymorphism, when the VVTT genotype was taken as the baseline risk, we found significant heterogeneity in the VITM genotype $\left(P_{Q}<0.1\right.$, $\left.I^{2}=69.2 \%\right)$. When the $249 \mathrm{~V}-280 \mathrm{~T}$ haplotype was taken as the baseline risk, we showed obvious heterogeneity in the 249I$280 \mathrm{M}$ haplotype $\left(P_{\mathrm{Q}}<0.1, I^{2}=82.2 \%\right.$; Table 3$)$.

3.7. Publication Bias. Begg's funnel plot and Egger's regression test were performed to assess potential publication bias. For the CX3CR1 T280M polymorphism, visual inspection of the funnel plot (Figure 4(a)) displays symmetrical distribution of OR estimations, suggesting no publication bias. In addition, the results of Egger's regression test also provided evidence for no publication bias (TM versus TT, $P>0.05$ for all genetic models). For the CX3CR1 V249I polymorphism, no obvious asymmetry was observed in any genetic model according to the visual assessment of funnel plot (Figure 4(b)). The results of Egger's regression test did not provide any statistical evidence for publication bias (VI versus $\mathrm{VV}, P>0.05$ for all genetic models).

\section{Discussion}

In this study, twenty-five articles involving the associations between the CX3CR1 polymorphisms and AS risk were included in the final meta-analysis. According to the GRADE approach, the quality of the evidence of CX3CR1 T280M polymorphism was very low in dominant model and allelic model, moderate in recessive model and additive model, and low in codominant model (Table S1). For the CX3CR1 V249I polymorphism, GRADE suggested that the quality of the evidence was moderate in recessive model, codominant model, and additive model, low in dominant model, and very low in allelic model (Table S1).

The overall findings showed that there was no association between the CX3CR1 polymorphisms and the risk of AS in five genetic models. To make a more comprehensive 
TABLE 4: Estimated haplotype frequencies and linkage disequilibrium analysis of the included studies.

\begin{tabular}{lcccccccccc}
\hline \multirow{2}{*}{ First author } & \multicolumn{3}{c}{ Estimated haplotype frequencies (cases) } & \multicolumn{3}{c}{ Estimated haplotype frequencies (controls) } & \multirow{2}{*}{$D^{\prime \text { a }}$} & $r^{2}{ }^{\text {a }}$ \\
& V249 T280 & V249 M280 & I249 T280 & I249 M280 & V249 T280 & V249 M280 & I249 T280 & I249 M280 & \\
\hline McDermott [13, 14] & 0.7817 & 0 & 0.0838 & 0.1345 & 0.7042 & 0 & 0.0986 & 0.1972 & 1 & 0.5848 \\
Moatti [15] & 0.7980 & 0 & 0.0993 & 0.1026 & 0.7149 & 0 & 0.1345 & 0.1506 & 1 & 0.4445 \\
Gugl [8] & 0.7317 & 0 & 0.0904 & 0.1778 & 0.7416 & 0 & 0.0706 & 0.1879 & 1 & 0.6638 \\
McDermott [13, 14] & 0.7304 & 0 & 0.1324 & 0.1373 & 0.7018 & 0 & 0.1157 & 0.1825 & 1 & 0.5253 \\
Ghilardi [7] & 0.7407 & 0 & 0.1481 & 0.1111 & 0.7255 & 0 & 0.1225 & 0.1520 & 1 & 0.4736 \\
Hattori [44] & 0.9318 & 0.0256 & 0.0065 & 0.0361 & 0.9444 & 0.0082 & 0.0033 & 0.0441 & 0.9270 & 0.7743 \\
Niessner [17, 18] & 0.7332 & 0.0016 & 0.1113 & 0.1540 & 0.7564 & 0 & 0.0998 & 0.1439 & 1 & 0.5217 \\
Apostolakis [6, 43] & 0.7214 & 0 & 0.1500 & 0.1286 & 0.7000 & 0 & 0.1212 & 0.1788 & 1 & 0.508 \\
An [24] & 0.8241 & 0 & 0.0833 & 0.0926 & 0.7187 & 0 & 0.1250 & 0.1562 & 1 & 0.4733 \\
Nassar-a [16] & 0.7067 & 0.0171 & 0.1440 & 0.1321 & 0.6544 & 0 & 0.1376 & 0.2081 & 1 & 0.4974 \\
Nassar-b [16] & 0.6250 & 0.0203 & 0.1622 & 0.1925 & 0.6544 & 0 & 0.1376 & 0.2081 & 1 & 0.4974 \\
Zhao [23] & 0.7138 & 0 & 0.1478 & 0.1384 & 0.6729 & 0 & 0.1353 & 0.1918 & 1 & 0.4882 \\
Singh-a, b [21, 22] & 0.7559 & 0.0542 & 0.1402 & 0.0497 & 0.6967 & 0 & 0.1050 & 0.1983 & 1 & 0.5682 \\
Singh-c [21, 22] & 0.7739 & 0.0413 & 0.1304 & 0.0543 & 0.6967 & 0 & 0.1050 & 0.1983 & 1 & 0.5682 \\
\hline Total & 0.7477 & 0.0091 & 0.1120 & 0.1312 & 0.7224 & 0.0006 & 0.1050 & 0.1720 & 0.9950 & 0.5394 \\
\hline
\end{tabular}

${ }^{\mathrm{a}} \mathrm{D}^{\prime}$ and $r^{2}$ statistics refer to control groups.

analysis, subgroup analyses were performed based on ethnicity, atherosclerotic disease, and source of controls. For the CX3CR1 T280M polymorphism, Apostolakis et al. [43] showed that the $280 \mathrm{M}$ allele carriers reduced the risk of $\mathrm{CAD}$ in heterozygote state. In the present study, we also found atheroprotective effect to $\mathrm{AS}$ in the CAD group. In addition, we found that the $280 \mathrm{M}$ allele carriers increased the risk of ICVD in homozygote state. For the CX3CR1 V249I polymorphism, significant associations were found between this variant and the atheroprotective effect on AS in CAD and PB groups. These results suggested that the 249I allele carriers reduced the risk of $\mathrm{CAD}$ in heterozygote state. The above statistical results were based on Bonferroni correction to control for the multiple testing in view of under investigation.

For the combined genotype, the VITM and IITM genotypes played an atheroprotective effect on AS. The combined VVTM genotype was more common in the cases than in the controls; however, the rarity of the VVTM genotype makes any conclusion rather unsafe. LD analysis indicated a strong association between T280M and V249I, and a protective role of the 249I-280M haplotype was also observed in the control population compared to case subjects. No association was observed between the 249I-280T haplotype and the susceptibility to AS. These results were consistent with those of a previous study [43].

Considering the studies without HWE or with low NOS score may influence the overall results, subsequent sensitivity analyses restricted to the studies with HWE or high NOS score were performed, but no corresponding pooled OR was materially altered in the dominant, recessive, codominant, additive, and allelic models. These results suggested that the studies without HWE or low score should not be considered as a factor influencing the overall results.

Heterogeneity of the included studies is the most important drawback when the genotypic data were analyzed, either for the CX3CR1 T280M or for the CX3CR1 V249I polymorphism. Heterogeneity should not be ignored and should be carefully factored in the interpretation of the final results. For the CX3CR1 T280M and V249I polymorphisms, the heterogeneity can partly be explained by the ethnicity (Caucasians and Asian), subtype of atherosclerotic diseases (CAD, CAA, and ICVD), and source of control (hospitalbased and population-based).

However, some limitations of this meta-analysis should be acknowledged. Firstly, there was significant heterogeneity in this meta-analysis. Heterogeneity may affect the precision of overall results, despite the use of appropriate meta-analytic techniques with random-effects model. Secondly, in the subgroup analyses, the sample sizes in some subgroup, such as the PAD and CAA groups of the CX3CR1 T280M and V249I polymorphisms, were relatively small, not having enough statistical power to explore the real association. Thirdly, AS is a complex disease and involves potential interactions of geneenvironment. However, many eligible studies included in this meta-analysis did not consider the environmental factors. Therefore, studies with larger sample sizes and better design are needed.

\section{Conclusion}

The present meta-analysis suggested that the CX3CR1 280M and 249I allele carriers had atheroprotective roles on AS in heterozygote state, and the $280 \mathrm{M}$ allele carriers were associated with the susceptibility to AS in homozygote state. The combined genotypes of VITM and IITM also had atheroprotective roles on AS. Consequently, this effect may be attributed to the haplotype of 249I-280M. However, the results should be interpreted with caution because of its limitations. Further studies with large sample size, especially with the consideration of gene-gene and gene-environment interactions, are needed to confirm our findings. 


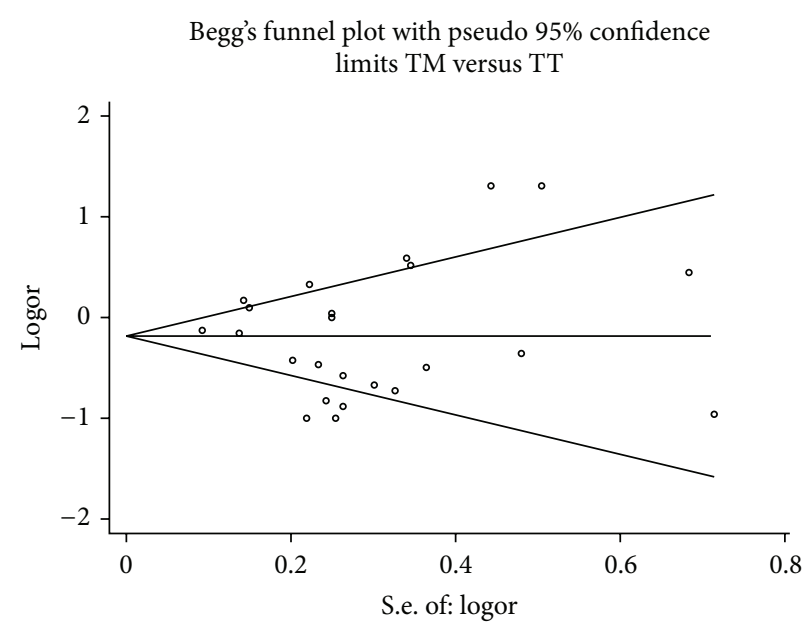

(a)

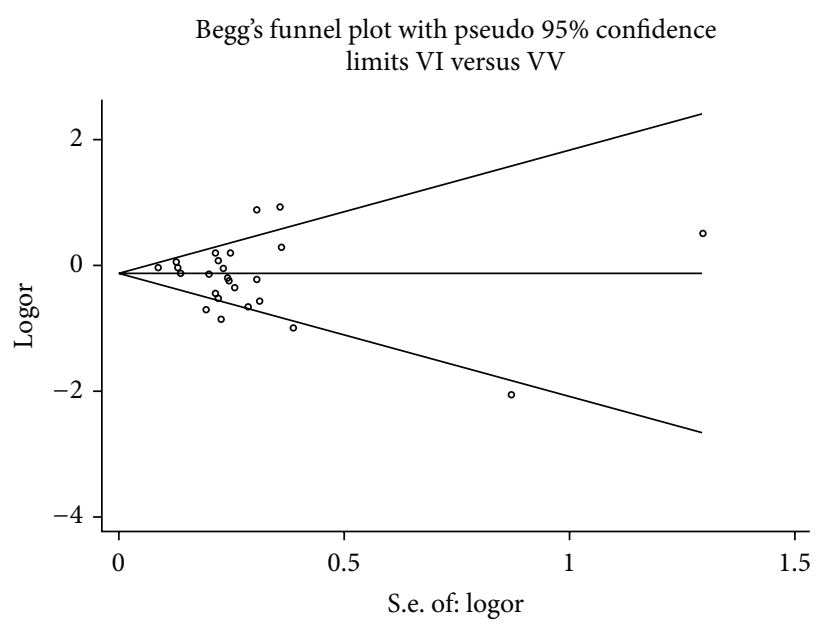

(b)

FIGURE 4: Begg's funnel plots for CX3CR1 T280M and V249I polymorphisms and AS risk. (a) TM versus TT for T280M polymorphism; (b) VI versus VV for V249I polymorphism.

\section{Conflict of Interests}

The authors declare that there is no conflict of interests regarding the publication of this paper.

\section{Acknowledgment}

This study was supported by the National Natural Science Foundation of China (no. 30960130).

\section{References}

[1] V. L. Roger, A. S. Go, D. M. Lloyd-Jones et al., "Heart disease and stroke statistics-2011 update: a report from the American Heart Association," Circulation, vol. 123, pp. e18-e209, 2011.

[2] P. D. Zende, M. P. Bankar, P. S. Kamble, and A. A. Momin, "Apolipoprotein e gene polymorphism and its effect on plasma lipids in arteriosclerosis," Journal of Clinical and Diagnostic Research, vol. 7, pp. 2149-2152, 2013.

[3] A. Ekmekçi, KS. Ozcan, B. Güngör et al., "The relationship between endothelial nitric oxide synthase $4 \mathrm{a} / 4 \mathrm{~b}$ gene polymorphism and premature coronary artery disease," Acta Cardiology, vol. 68 , pp. 464-468, 2013.

[4] M. García-Bermúdez, R. López-Mejías, F. Genre et al., "SMAD3 rs17228212 gene polymorphism is associated with reduced risk to cerebrovascular accidents and subclinical atherosclerosis in anti-CCP negative Spanish rheumatoid arthritis patients," PLoS ONE, vol. 8, Article ID e77695, 2013.

[5] M. Al-Najai, P. Muiya, A. I. Tahir et al., "Association of the angiotensinogen gene polymorphism with atherosclerosis and its risk traits in the Saudi population," BMC Cardiovascular Disorders, vol. 13, article 17, 2013.

[6] S. Apostolakis, S. Baritaki, G. E. Kochiadakis, N. E. Igoumenidis, D. Panutsopulos, and D. A. Spandidos, "Effects of polymorphisms in chemokine ligands and receptors on susceptibility to coronary artery disease," Thrombosis Research, vol. 119, no. 1, pp. 63-71, 2007.
[7] G. Ghilardi, M. L. Biondi, O. Turri, E. Guagnellini, and R. Scorza, "Internal carotid artery occlusive disease and polymorphisms of fractalkine receptor CX3CR1: a genetic risk factor," Stroke, vol. 35, no. 6, pp. 1276-1279, 2004.

[8] A. Gugl, W. Renner, G. Seinost et al., “Two polymorphisms in the fracalkine receptor CX3CR1 are not associated with peripheral arterial disease," Atherosclerosis, vol. 166, no. 2, pp. 339-343, 2003.

[9] M. Kimouli, S. Miyakis, P. Georgakopoulos, E. Neofytou, A. D. Achimastos, and D. A. Spandidos, "Polymorphisms of fractalkine receptor $C X 3 C R 1$ gene in patients with symptomatic and asymptomatic carotid artery stenosis," Journal of Atherosclerosis and Thrombosis, vol. 16, no. 5, pp. 604-610, 2009.

[10] E. Lavergne, J. Labreuche, M. Daoudi et al., "Adverse associations between CX3CR1 polymorphisms and risk of cardiovascular or cerebrovascular disease," Arteriosclerosis, Thrombosis, and Vascular Biology, vol. 25, no. 4, pp. 847-853, 2005.

[11] Z. Li, S. Luo, F. Wang, J. Zhen, H. Sun, and C. Guo, "An association study between polymorphisms of the Fractalkine receptor gene, CX3CR1, and cerebral infarction in the Han Chinese population," Journal of the Neurological Sciences, vol. 320, no. 1-2, pp. 12-15, 2012.

[12] E. M. Matzhold, O. Trummer, G. Grünbacher et al., "Association of polymorphisms in the chemokine receptor CX3CR1 gene with coronary artery disease," Cytokine, vol. 47, no. 3, pp. 224227, 2009.

[13] D. H. McDermott, J. P. J. Halcox, W. H. Schenke et al., "Association between polymorphism in the chemokine receptor CX3CR1 and coronary vascular endothelial dysfunction and atherosclerosis," Circulation Research, vol. 89, no. 5, pp. 401-407, 2001.

[14] D. H. McDermott, A. M. Fong, Q. Yang et al., "Chemokine receptor mutant CX3CR1-M280 has impaired adhesive function and correlates with protection from cardiovascular disease in humans," The Journal of Clinical Investigation, vol. 111, no. 8, pp. 1241-1250, 2003

[15] D. Moatti, S. Faure, F. Fumeron et al., "Polymorphism in the fractalkine receptor CX3CR1 as a genetic risk factor for 
coronary artery disease," Blood, vol. 97, no. 7, pp. 1925-1928, 2001.

[16] B. A. Nassar, A. A. Nanji, T. P. Ransom et al., "Role of the fractalkine receptor CX3CR1 polymorphisms V249I and T280M as risk factors for early-onset coronary artery disease in patients with no classic risk factors," Scandinavian Journal of Clinical and Laboratory Investigation, vol. 68, no. 4, pp. 286-291, 2008.

[17] A. Niessner, R. Marculescu, A. Haschemi et al., "Opposite effects of CX3CR1 receptor polymorphisms V249I and T280M on the development of acute coronary syndrome. A possible implication of fractalkine in inflammatory activation," Thrombosis and Haemostasis, vol. 93, no. 5, pp. 949-954, 2005.

[18] A. Niessner, R. Marculescu, H. Kvakan et al., "Fractalkine receptor polymorphisms V2491 and T280M as genetic risk factors for restenosis," Thrombosis and Haemostasis, vol. 94, no. 6, pp. 1251-1256, 2005.

[19] S. Pucci, P. Mazzarelli, M. J. Zonetti et al., "CX3CR1 receptor polymorphisms, Th1 cell recruitment, and acute myocardial infarction outcome: looking for a link," BioMed Research International, vol. 2013, Article ID 451349, 9 pages, 2013.

[20] D. L. S. Rios, S. M. Callegari-Jacques, and M. H. Hutz, "Endothelial nitric oxide synthase and fractalkine chemokine receptor polymorphisms on angiographically assessed coronary atherosclerosis," Clinica Chimica Acta, vol. 362, no. 1-2, pp. 138146, 2005.

[21] N. Singh, H. Rai, N. Sinha, S. Kumar, C. M. Pandey, and S. Agrawal, "Association of V249I and T280M polymorphisms in the chemokine receptor CX3CR1 gene with early onset of coronary artery disease among North Indians," Genetic Testing and Molecular Biomarkers, vol. 16, no. 7, pp. 756-760, 2012.

[22] N. Singh, N. Sinha, S. Kumar, C. M. Pandey, and S. Agrawal, "Polymorphism in chemokine receptor genes and risk of acute myocardial infarction in North Indian population," Molecular Biology Reports, vol. 39, no. 3, pp. 2753-2759, 2012.

[23] R. Zhao, Y. Wang, R. Shen, and Y. Sun, "Relationship between CX3CR1 genetic polymorphism and carotid atherosclerosis," Artificial Cells, Blood Substitutes, and Biotechnology, vol. 38, no. 1, pp. 19-23, 2010.

[24] Z. An, L. Bi, L. Y. Zheng, and J. Sun, "Association between polymorphism in chemokine receptor CX3CR1 and coronary atherosclerosis," Journal of Dalian Medical University, vol. 30, pp. 314-317, 2008.

[25] X. Peng, Z. B. Zhang, L. Tang, and Y. Zhou, "Study on chemokine receptor CX3CR1 gene T280M polymorphism in patients with ischemic cerebrovascular disease," Journal of Clinical Neurology, vol. 21, pp. 329-332, 2008.

[26] L. Shi, L. Sun, P. Gu et al., "Relationship between chemokine receptor CX3CR1 gene polymorphism with cerebral infarction of North China population," Journal of Apoplexy and Nervous Diseases, vol. 29, pp. 434-438, 2012.

[27] L. Wu, L. Jia, X. Guo, D. Liu, and H. Sun, "A study on CX3CR1 gene polymophism of the Han nationality people in Baotou," Journal of Baotou Medical College, vol. 28, pp. 9-11, 2012.

[28] H. K. Xiao, L. Z. Jin, G. T. Xie, J. Chen, X. C. Chen, and M. $\mathrm{T}$. Xu, "A study on association of Fractalkine receptor CX3CR1 gene polymorphism to coronary heart disease," Chinese Journal of Arteriosclerosis, vol. 18, pp. 993-995, 2010.

[29] Z. B. Zhang, X. Peng, and L. Tang, "Research on V249I polymorphisms of chemokine receptor CX3CR1 gene in patients with ischemic cerebrovascular disease," Shandong Medical Journal, vol. 48, pp. 15-17, 2008.
[30] Y. L. Zhang, W. Yu, and B. Fang, "Association study between the polymorphisms of fractalkine receptor CX3CR1 and cerebral infarction," China Journal of Modern Medicine, vol. 20, pp. 48, 2010.

[31] M. Zheng and C. Zhang, "The association of CX3CR1 polymorphisms with carotid artery intima-media thickness," Acta Academiae Medicinae Qingdao Universitatis, vol. 44, pp. 216219, 2008.

[32] A. S. Jaipersad, G. Y. Lip, S. Silverman, and E. Shantsila, “The role of monocytes in angiogenesis and atherosclerosis," Journal of the American College of Cardiology, vol. 63, pp. 1-11, 2014.

[33] J. Frostegård, "Immunity, atherosclerosis and cardiovascular disease," BMC Medicine, vol. 11, article 117, 2013.

[34] A. Ghattas, H. R. Griffiths, A. Devitt, G. Y. Lip, and E. Shantsila, "Monocytes in coronary artery disease and atherosclerosis: where are we now?" Journal of the American College of Cardiology, vol. 62, no. 17, pp. 1541-1551, 2013.

[35] C. Weber, A. Schober, and A. Zernecke, "Chemokines: key regulators of mononuclear cell recruitment in atherosclerotic vascular disease," Arteriosclerosis, Thrombosis, and Vascular Biology, vol. 24, no. 11, pp. 1997-2008, 2004.

[36] H. Liu and D. Jiang, "Fractalkine/CX3CR1 and atherosclerosis," Clinica Chimica Acta, vol. 412, no. 13-14, pp. 1180-1186, 2011.

[37] J. F. Bazan, K. B. Bacon, G. Hardiman et al., "A new class of membrane-bound chemokine with a CX3C motif," Nature, vol. 385, no. 6617, pp. 640-642, 1997.

[38] D. R. Greaves, T. Häkkinen, A. D. Lucas et al., "Linked chromosome 16q13 chemokines, macrophage-derived chemokine, fractalkine, and thymus- and activation-regulated chemokine, are expressed in human atherosclerotic lesions," Arteriosclerosis, Thrombosis, and Vascular Biology, vol. 21, no. 6, pp. 923-929, 2001.

[39] B. W. C. Wong, D. Wong, and B. M. McManus, "Characterization of fractalkine (CX3CL1) and CX3CR1 in human coronary arteries with native atherosclerosis, diabetes mellitus, and transplant vascular disease," Cardiovascular Pathology, vol. 11, no. 6, pp. 332-338, 2002.

[40] C. Combadière, S. Potteaux, J. Gao et al., "Decreased atherosclerotic lesion formation in CX3CR1/apolipoprotein E double knockout mice," Circulation, vol. 107, no. 7, pp. 1009-1016, 2003.

[41] P. Lesnik, C. A. Haskell, and I. F. Charo, "Decreased atherosclerosis in $\mathrm{CX}_{3} \mathrm{CR}^{-1-}$ mice reveals a role for fractalkine in atherogenesis," The Journal of Clinical Investigation, vol. 111, no. 3, pp. 333-340, 2003.

[42] N. Saederup, L. Chan, S. A. Lira, and I. F. Charo, "Fractalkine deficiency markedly reduces macrophage accumulation and atherosclerotic lesion formation in CCR2-/- mice: evidence for independent chemokine functions in atherogenesis," Circulation, vol. 117, no. 13, pp. 1642-1648, 2008.

[43] S. Apostolakis, V. Amanatidou, E. G. Papadakis, and D. A. Spandidos, "Genetic diversity of CX3CR1 gene and coronary artery disease: new insights through a meta-analysis," Atherosclerosis, vol. 207, no. 1, pp. 8-15, 2009.

[44] H. Hattori, D. Ito, N. Tanahashi et al., “T280M and V249I polymorphisms of fractalkine receptor CX3CR1 and ischemic cerebrovascular disease," Neuroscience Letters, vol. 374, no. 2, pp. 132-135, 2005.

[45] D. Moher, A. Liberati, J. Tetzlaff, and D. G. Altman, "Preferred reporting items for systematic reviews and meta-analyses: the PRISMA statement," International Journal of Surgery, vol. 8, no. 5, pp. 336-341, 2010. 
[46] G. A. Wells, B. Shea, D. O'Connell et al., “The Newcastle-Ottawa Scale (NOS) for assessing the quality of nonrandomised studies in meta-analyses," Ottawa Health Research Institute, http://www.ohri.ca/programs/clinical_epidemiology/oxford .asp.

[47] G. H. Guyatt, A. D. Oxman, G. E. Vist et al., "GRADE: an emerging consensus on rating quality of evidence and strength of recommendations," British Medical Journal, vol. 336, no. 7650, pp. 924-926, 2008.

[48] T. R. Gaunt, S. Rodríguez, and I. N. M. Day, "Cubic exact solutions for the estimation of pairwise haplotype frequencies: implications for linkage disequilibrium analyses and a web tool "CubeX"', BMC Bioinformatics, vol. 8, article 428, 2007.

[49] J. P. T. Higgins, S. G. Thompson, J. J. Deeks, and D. G. Altman, "Measuring inconsistency in meta-analyses," British Medical Journal, vol. 327, no. 7414, pp. 557-560, 2003.

[50] C. S. Berkey, D. C. Hoaglin, F. Mosteller, and G. A. Colditz, "A random-effects regression model for meta-analysis," Statistics in Medicine, vol. 14, no. 4, pp. 395-411, 1995. 


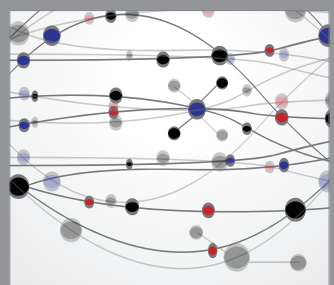

The Scientific World Journal
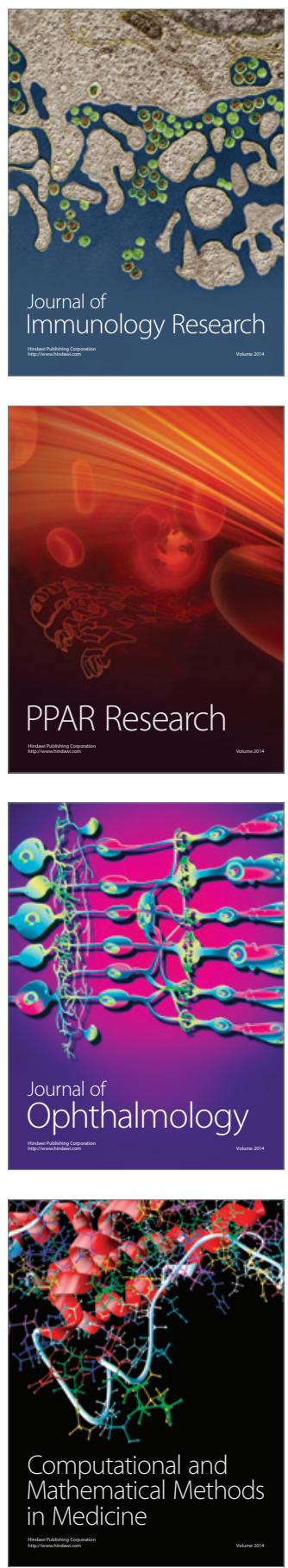

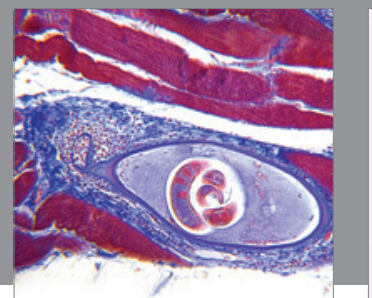

Gastroenterology

Research and Practice
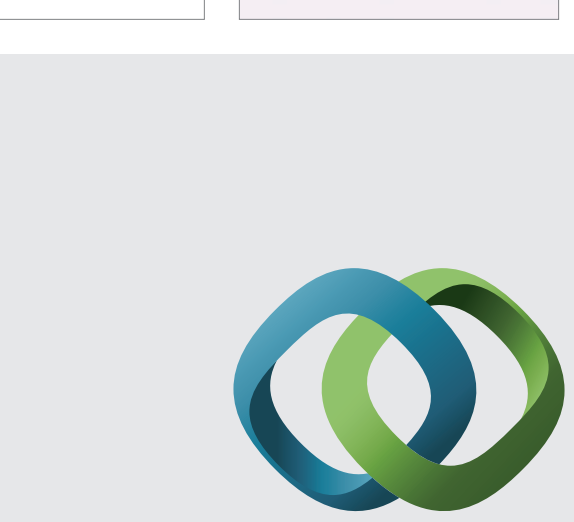

\section{Hindawi}

Submit your manuscripts at

http://www.hindawi.com
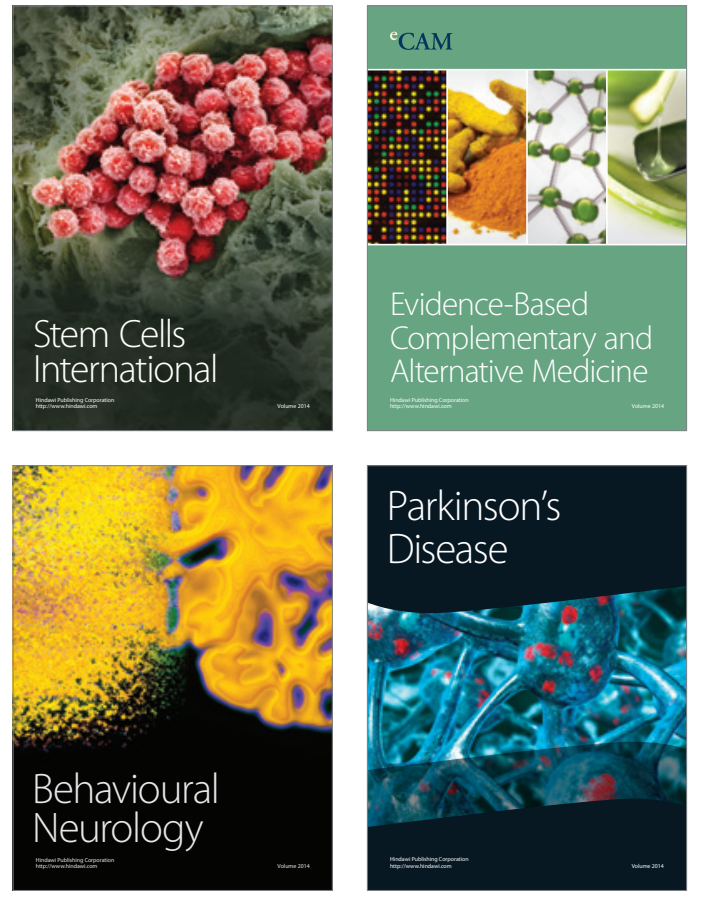
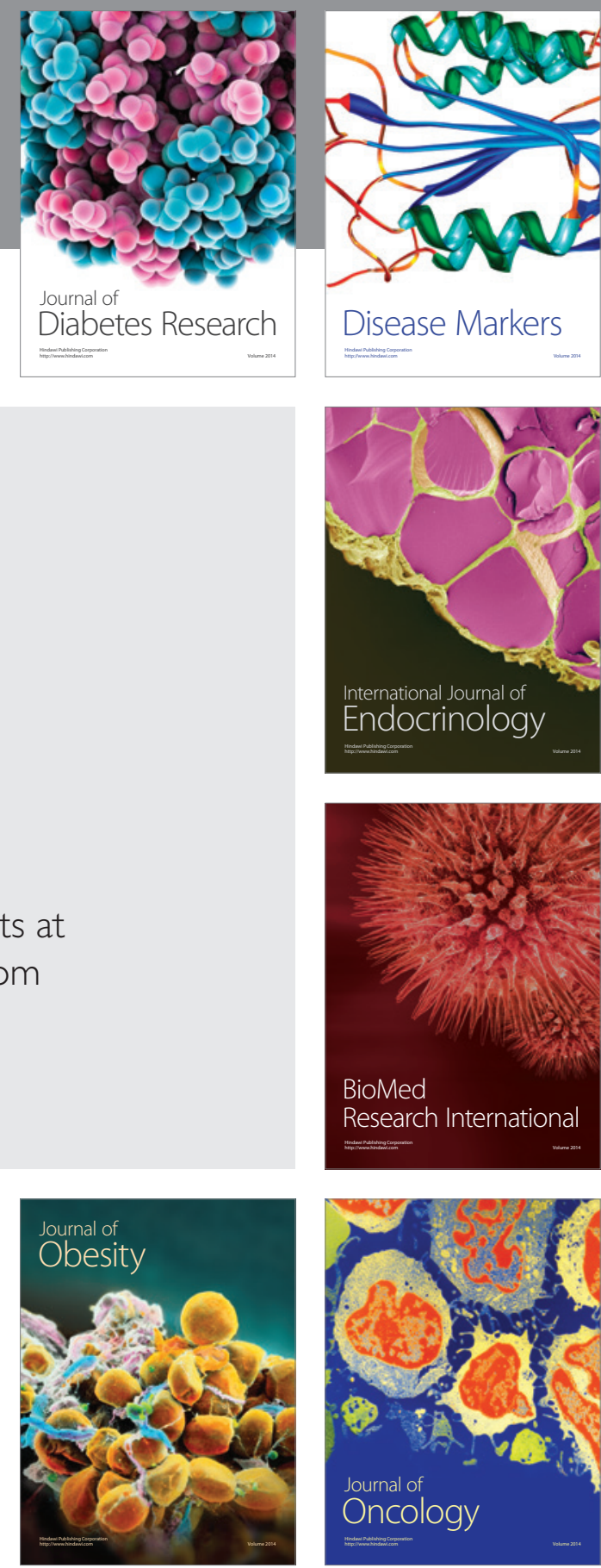

Disease Markers
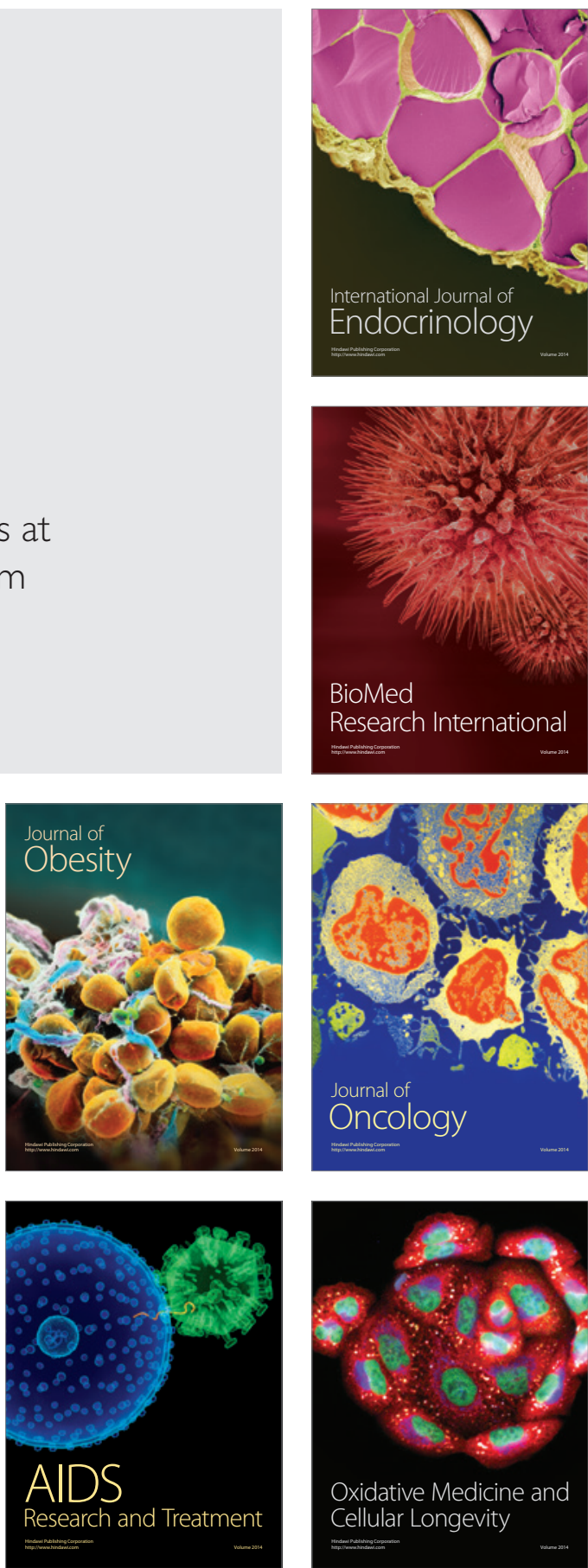